\title{
El bien-ser-estar humano, reseña del número 256 de la revista Anthropos
}

\author{
Carlos Barba Solano
}

Esta reseña tiene como objetivo analizar el número 256 de la revista Anthropos. Cuadernos de cultura crítica y conocimiento, coordinado por Julio Boltvinik (2020), cuyo tema central es la presentación de enfoques encontrados sobre el bien-ser-estar humano. Este número monográfico está integrado por tres artículos, una traducción a cargo de Israel Grande-García y Julio Boltvinik del prefacio e introducción del libro Homo Prospectur, publicado por Oxford University Press en 2016, cuyos autores son Martin Seligaman, Peter Railton, Roy Baumeister y Chandra Sripada; una reseña sobre el mismo libro realizada de Aura Ponce de León; así como la reedición de dos capítulos del libro pionero en el campo de la economía conductista intitulado The Joyless Economy publicado por Tibor Scitovsky en $1976 .{ }^{1}$ En esta reseña voy a concentrarme en presentar los tres artículos solicitados para integrar el núcleo de esta propuesta, el primero elaborado por Ian Gough, el segundo por Mariano Rojas y el tercero por Carlos Arellano y Julio Boltvinik.

I. Que de acuerdo con Julio Boltvinik es el fundador de la economía conductista. Este libro fue traducido al español por el FCE en 1986 bajo el título Frustración de la riqueza. La satisfacción humana y la insatisfacción del consumidor.

Fecha de recepción: $X$ de $x x x x x$ de 202X. Fecha de aceptación: $X$ de $x x x x x$ de 202X.

-Profesor-investigador en la Universidad de Guadalajara. Miembro del SNI, nivel III.

Boltvinik, Julio (2020). Anthropos. Cuadernos de cultura crítica y conocimiento. vol(256), $X X-X X$. 
En el artículo intitulado "De cara al colapso climático, las necesidades humanas son centrales para el bien-estar sustentable", Ian Gough, profesor visitante en el Center for the Analysis of Social Exclusion, de la London School of Economics, sostiene justamente que frente al colapso climático que amenaza con producirse como resultado del calentamiento global y la descomposición ecológica que caracterizan nuestra época y que pone en un predicamento el bienestar humano a escala global no sólo hoy sino en el futuro, el bienestar debe ser abordado a partir de una concepción de las necesidades humanas básicas, porque ni la tesis de la soberanía del consumidor, ni la de la felicidad como medida de bienestar, ni el relativismo cultural posmoderno, poscolonial y/o postestructuralista, ni siquiera el enfoque de las capacidades de Amartya Sen son eficaces para realizar esa tarea.

Gough aborda y critica ampliamente y con profundidad la teoría de la satisfacción de las preferencias de los consumidores, emanada de la economía del bienestar, que atribuye el bienestar a la satisfacción de apetencias ilimitadas, sin realizar una evaluación moral, lógica y práctica de su utilidad, ni considerar sus consecuencias para el futuro de la humanidad. Al modelo del Homo economicus Gough opone una afirmación que tiene raíces teóricas, antropológicas, sociológicas e histórico/evolutivas: que cada individuo no sólo es movido por el propio interés sino por la preocupación por los demás, por la cooperación, por el trato equitativo y el repudio a quienes violan las normas de equidad.

Gough crítica a la economía neoclásica por aplanar la distinción moral entre deseos individuales y necesidades sociales, que equiparan el deseo "del epicúreo que quiere más sazón" con la necesidad "del niño hambriento que quiere un poco de agua”. Así, ante la amenaza de colapso futuro que enfrenta la humanidad, Gough sostiene la flagrante inadecuación de la satisfacción de preferencias para 
garantizar al bienestar de generaciones futuras y contrapone a la transmisión intergeneracional de un acervo de capital para mantener un determinado nivel de consumo a los herederos, la meta y la obligación de sustentabilidad, prerrequisito para pensar el bienestar a una escala global e intergeneracional.

En el caso de la teoría de la felicidad y sus métricas, Gough considera que ésta enfrenta problemas semejantes a los de la teoría utilitarista, por varias razones, entre ellas: su menosprecio por la tendencia omnipresente en la humanidad de ajustar las expectativas a la realidad, su tendencia a reportar el bienestar a partir de valores individualistas. Al abordar la teoría de las capabilities de Sen y su tentativa de concebir el bienestar humano en términos de libertades, oportunidades sustantivas y capacidades basadas en los functionings de las personas (aquello que son capaces de lograr cuando se lo proponen), Gough reconoce un avance significativo en la construcción del índice de desarrollo humano, frente a la visión del Homo economicus y la métrica del PIB. Sin embargo, señala que Sen no logra ofrecer una visión universal de las capacidades comunes y los functionings básicos y carece de una visión que garantice la protección de las generaciones futuras.

Gough nos recuerda que las necesidades humanas son objetivas, plurales (no pueden sumarse o reducirse a una sola), transgeneracionales, no son sustituibles entre sí y aunque se pueden saciar, de no ser satisfechas causan daños objetivos porque afectan la salud, evitan que las personas puedan participar en la sociedad e impiden la posibilidad de reflexionar críticamente acerca de las condiciones sociales. A las necesidades universales las denomina necesidades básicas (salud, autonomía y participación social efectiva), pero considera que sus satisfactores no son universales debido a la existencia de múltiples contextos culturales. 
Un elemento fundamental de este trabajo es la propuesta de Gough de articular el desarrollo sustentable y las necesidades humanas, que implica definir al primero como aquel que satisface las necesidades del presente sin poner en peligro la posibilidad de que las generaciones del futuro puedan satisfacer las suyas.

Un enfoque como éste plantea el bienestar como una construcción transdisciplinar y exige abordarlo como un objeto que rebasa el ámbito de acción de las políticas económica, social o ambiental consideradas de manera aislada y exige concebir, en el marco de la economía política, el diseño de un mix del bienestar que no considere como riesgos sociales sólo los que se relacionan con la pobreza y las desigualdades sociales en el presente, sino aquellos que imposibilitan el bienestar de las generaciones futuras, tema absolutamente crucial.

En el artículo titulado "El bienestar como vivencia. Consideraciones conceptuales para el estudio del bienestar", Mariano Rojas, ex profesor de la FLACso México, actualmente adscrito a la Universidad Internacional de La Rioja en España, realiza una defensa del enfoque del bienestar subjetivo y una crítica muy fuerte a los enfoques del bienestar objetivo. El choque entre esta visión y la de Gough es inevitable porque Rojas concibe el bienestar, no a partir de las necesidades humanas básicas, universales y objetivas, sino como una vivencia subjetiva, experimentada por las personas; valga decir, por los individuos.

El punto de partida de esta perspectiva es la existencia de una esencia humana, de una condición humana, que no son discutidas, pero se traducen en un rechazo implícito a la tentativa sociológica de ubicar a los seres humanos en contextos sociales, culturales, económicos e históricos específicos. ${ }^{2}$ Sin embargo, para la sociología las personas,

2. Giddens (1994) señala que nuestras expectativas y deseos no son los mismos que los de otras culturas: enamorarse es una experiencia situada históricamente,

\section{2}


los individuos, no se explican por sí mismos, son el resultado de complejos procesos de socialización; sus ideas acerca de las cosas, su autoconcepción, su percepción sobre la propia vida están mediadas por discursos, prácticas y representaciones sociales. ${ }^{3}$

Tanto en el ámbito de la filosofía como en el de la sociología fenomenológica, que tiene como objeto central "la experiencia”, autores como Edmund Husserl (1984), Alfred Schütz (1972) y Peter Berger y Thomas Luckmann (1968) reconocen que el sentido nace de la articulación entre los sujetos y el mundo y es de carácter intersubjetivo.

Schütz concebía la significatividad como el resultado de un encuentro entre la corriente interna de la conciencia y lo que ocurre en el mundo de la vida, con la mediación de las experiencias previas, propias y ajenas acumuladas en "acervos de conocimiento" disponibles en el lenguaje. Para él, la "actitud natural" es en realidad social, porque para hacer comprensible la realidad recurrimos a tipificaciones, de carácter valorativo e interpretativo, acerca de objetos, personas o acontecimientos sociales que permiten ubicar, clasificar e interpretar las vivencias de las personas, aun las propias.

Berger y Luckmann sostenían, como lo hizo más tarde Bourdieu, que los sujetos humanos interiorizan el mundo objetivo a través de los procesos de socialización primaria (durante la niñez) y de manera intersubjetiva a través de la

desear formar un hogar o buscar una realización personal y sexual en una relación, al igual que fracasar o lograrlo, son elementos propios de la cultura occidental; estos ejemplos ilustran que lo que percibimos como natural es construido social e históricamente.

3. La sociología desde su fundación ha puesto en duda la pertinencia de la idea de naturaleza humana porque considera que nuestras experiencias están condicionadas por profundas alteraciones históricas, estructurales y por instituciones concretas. 
socialización en distintos submundos a lo largo de la vida y que en ese proceso el lenguaje es el factor clave. ${ }^{4}$

A pesar de ello, Rojas argumenta que las experiencias de bienestar son fenómenos individuales, generalizables a todos los seres humanos y son de cuatro tipos: evaluativas, afectivas, sensoriales y de flujo. Las evaluativas son binarias: logros o fracasos respecto a metas, aspiraciones y propósitos vitales. Las afectivas también lo son: gozo o sufrimiento. El patrón continúa en el caso de las sensoriales: placer o dolor, derivados de los sentidos. Finalmente, las experiencias de flujo generan recompensas o situaciones desagradables en sí mismas e implican un involucramiento intenso pero breve con acciones humanas, como bailar, escuchar una sinfonía o conversar con alguien. Rojas señala que los cuatro tipos de experiencias están interrelacionadas de manera compleja y que pueden experimentarse de manera conjunta, de manera armónica o conflictiva. En todos los casos el autor subraya que no deben confundirse las vivencias con los factores que las generan, porque un mismo evento puede disparar diferentes y opuestas experiencias evaluativas.

Este enfoque considera que el bienestar subjetivo puede medirse a partir de la evaluación que los individuos realizan sobre sus experiencias, respondiendo a preguntas directas y simples que pueden sintetizarse en escalas numéricas; este tema es muy controvertido, como queda claro en el artículo escrito por Arellano y Boltvinik.

Al final de la lectura de este trabajo, aunque no me parece que la propuesta de este enfoque de referirse a los puntos de vista de los individuos sobre sus experiencias de bienestar sea irrelevante para confrontarlo con las ideas que los expertos tienen acerca del bienestar, me quedo con la impresión

4. Porque permite internalizar el mundo objetivo y los esquemas motivacionales e interpretativos construidos intersubjetivamente. Por otra parte, sobre el carácter histórico de nuestras experiencias. 
de que este enfoque rehúye pensar que todo individuo vive una biografía dentro de un contexto sociocultural y económico, que las experiencias de bienestar están formadas por la sociedad y forman parte de un impulso histórico. Esto es justamente lo que Wright Mills (1961) proponía como la tarea y la promesa de la imaginación sociológica: captar la historia, la biografía y las relaciones entre ambas dentro de la sociedad.

Considero que este enfoque sobre el bien-estar subjetivo deja de lado aspectos intersubjetivos, colectivos, relacionales e institucionales, cruciales para dar cuenta de fenómenos como las desigualdades sociales y la exclusión sociocultural, y para comprender los mayúsculos dilemas morales e históricos del presente, que condicionan las vivencias del bienestar.

Finalmente, en el artículo de Carlos Arellano, de la Universidad Autónoma de Zacatecas, y Julio Bolvinik, de El Colegio de México, se aborda el campo de los estudios sobre el bien-ser-estar a partir de su división en dos subcampos: el bien-ser-estar objetivo y el subjetivo. El abordaje del bienser-estar objetivo inicia con la critica que realiza Amartya Sen a la visión neoclásica del consumidor, que postula la maximización de la utilidad y rechaza la idea de utilidad marginal decreciente porque han incorporado el principio de no saciedad y defienden la relación lineal entre mayor consumo y más bienestar. Sen denomina a este enfoque como "el de la opulencia" porque correlaciona el crecimiento económico con la disponibilidad de ingreso de las familias y considera el crecimiento del PIB como equivalente al desarrollo, sin conceder importancia alguna a la desigualdad en la distribución del ingreso. En su visión extrema ese enfoque considera irrelevantes las comparaciones interpersonales de utilidad y también las cuestiones éticas, por ello recurre a la noción de óptimo de Pareto, que sostiene que sólo puede haber mejoría social cuando algunos son beneficiados, pero nadie es perjudicado (con una gran ironía, los autores

Reseñas No.82 
señalan que la derrota de la Alemania nazi no cumple con ese estándar, porque Hitler perdió).

Los autores de este texto reconocen el avance logrado por el enfoque de Sen (1999) sobre las capabilities-functionings cuya tesis fundamental es que los medios o los recursos son buenos por lo que le permiten ser o hacer a los seres humanos y el tipo de vida que les permiten elegir y vivir. Sin embargo, subrayan una serie de deficiencias en ese enfoque, entre ellas sobresale su incapacidad para refutar la idea de que el elemento constitutivo del bienestar es la satisfacción objetiva de las necesidades humanas. En ese terreno, señalan que Martha Nussbaum logra avanzar mucho más, al definir una lista de capacidades básicas innatas (hablar, amar, gratitud, razón práctica y trabajo) y de funciones que son centrales para la vida humana, así como un umbral específico en cada capacidad. Nussbaum (2000) considera que un ser humano es libre, digno, moldea su propia vida a través del uso de la razón práctica, la sociabilidad y la reciprocidad. Esas ideas son semejantes a las desarrolladas por Boltvinik en su visión sobre el florecimiento humano.

Arellano y Boltvinik defienden aspectos centrales del enfoque de satisfacción de necesidades, la teoría de necesidades humanas y van más allá con la teoría del florecimiento humano, porque éste no concibe a las personas como consumidores sino como seres humanos. Indican que la satisfacción de las necesidades implica realizar un propósito universal: evitar un daño a los humanos, lo que no ocurre en el caso de los deseos. Consideran fundamental la utilización de la perspectiva de Marx, recuperada por Márkus (1973), sobre la esencia humana que reivindica al trabajo libre como la actividad vital para satisfacer necesidades, porque posibilita la autoconciencia de los seres humanos y les confiere un carácter social e histórico.

En este marco, Ryan y Deci (2001) abordan el concepto de bienestar a partir de la teoría de la autodeterminación que 
identifica tres necesidades psicológicas básicas cuya satisfacción es necesaria para el bienestar en distintas etapas y a partir de una perspectiva transcultural, referidos a la realización de posibilidades intrínsecas de los seres humanos: autonomía, aptitud y sociabilidad. De acuerdo con esos autores el desarrollo del ser humano implica el desarrollo de sus necesidades y capacidades históricas, porque ello implica una humanización de las necesidades biológicas y la creación de nuevas necesidades, como el aprendizaje y las necesidades estéticas o científicas. Esto implica una fuerte ruptura entre el concepto de necesidades y los de apetencias y deseos, porque las necesidades objetivas se ligan al propósito universal de evitar un daño a los humanos, mientras los deseos no. Mientras los economistas ortodoxos restringen los satisfactores de apetencias a bienes y servicios, la teoría de las necesidades humanas identifica objetos, relaciones y actividades que son satisfactores de necesidades.

Bajo este nuevo enfoque resulta evidente el agotamiento de la legitimidad del economicismo, aunque siga vigente, por ello autores como Atkinson y Sen propusieron como una medida aproximada al bien-estar el PIB corregido con la equidad de su distribución, utilizando también el coeficiente de Gini (G) (PIB per cápita (1-G)), lo que implícitamente recupera la idea de utilidad marginal decreciente, porque quitar una $X$ cantidad de ingreso a las familias ricas y transferirlo a las pobres implica aumentar en una medida mucho mayor su bienestar, que la reducción del bienestar de las primeras.

Para estos enfoques el bienestar entraña el desarrollo de las fuerzas esenciales humanas (necesidades y capacidades), que tienden a la universalidad, a la libertad, creatividad y conciencia. La visión universalista entra en una fuerte contradicción con la alienación que caracteriza a la sociedad capitalista, que se traduce en una creciente unilateralidad individualista. Por ello, los autores indican que para lograr

Reseñas No.82 
el florecimiento humano hace falta el progreso social, vencer tanto la pobreza económica como la alienación, ya que la idea no es sólo satisfacer necesidades, sino desarrollarlas en su conjunto para concebir a un ser humano completo, porque en situaciones de pobreza predominan las necesidades fisiológicas, sin considerar el resto de ellas.

En la sección que analiza el enfoque del "Bien-ser-estar subjetivo" se afirma que en cierta forma este tipo de enfoque constituye un retorno al utilitarismo, porque concibe el bienestar como experiencia de felicidad de corto plazo o de satisfacción con la vida estable y de largo plazo, que pueden ser medidas, por ejemplo, utilizando encuestas, a partir de la propia valoración que los individuos hacen de sus vivencias, porque esta teoría asume que sólo ellos pueden juzgar su bienestar. El enfoque del bien-ser-estar subjetivo es problemático por diversas razones, entre ellas: porque consideran que los individuos son la única autoridad para juzgar qué tan apropiadas son sus apetencias y experiencias de bienestar, sin atreverse a formular ningún principio universal, ni juicio alguno de valor y por su pretensión de medir este tipo de bienestar a través del uso de escalas numéricas y de las respuestas directas de los individuos, a pesar de que las evidencias ponen en duda la validez y confiabilidad empírica de dichas medidas porque no necesariamente hay un entendimiento común sobre el significado de la felicidad o la satisfacción con la vida.

Sin embargo subrayan que, aunque se suele equiparar felicidad y bienestar subjetivo, una veta interesante que podría tender un puente entre el enfoque subjetivo y el objetivo es la concepción del bienestar eudaimónico, propuesta por Deci y Ryan (2001), que tiene como meta no el placer sino la realización del potencial humano (el "daimon" o verdadera naturaleza), lo que se asemeja al propósito del florecimiento humano. En esa tónica el artículo cierra

\section{8}


destacando la deseabilidad de una perspectiva integradora: bie-ser-nestar objetivo-subjetivo-florecimiento.

Sin duda, este número especial de la revista Anthropos. Cuadernos de cultura crítica y conocimiento realiza una contribución importante a la discusión integrada, en el contexto iberoamericano, de tres temáticas que deben ser abordadas de manera conjunta: la necesidad de abordar críticamente la perspectiva economicista del desarrollo, los dilemas que plantean el bien-ser-estar humano en términos objetivos y subjetivos y el futuro de la humanidad ligado a la sustentabilidad ambiental, por ello recomiendo ampliamente su lectura.

Berger, Peter, y Luckmann, Thomas. (1968). La construcción Bibliografía social de la realidad. Buenos Aires:Amorrortu Editores. Boltvinik, Julio. (Coord.). (2020). Anthropos. Cuadernos de cultura crítica y conocimiento, núm. 256, julio-septiembre. Barcelona: Siglo XXI Editores.

Giddens, Anthony. (1994). Sociología, problemas y perspectivas. Sociología (pP. 4l-59). Madrid:Alianza Editorial. Husserl, Edmund. (1984). Crisis de las ciencias europeas y la fenomenología trascendental. México: Folios Ediciones.

Markus, György. (1973). Marxismo y antropología. Barcelona: Grijalbo.

Mills, C.Wright. (196I). La promesa. La imaginación sociológica (pp. 23-43). México: Fondo de Cultura Económica.

Nussbaum, Martha. (2000). Women and human development: The capabilities approach. Cambridge: Cambridge University Press.

Ryan, y Deci. (200I). On Happiness and Human Potentials: A Review of Research on Hedonic and Eudaimonic Well-Being. Annual Review of Psychology, núm. 52, Pp. |4|-166.

Schütz, Alfred. (1972). Fenomenología del mundo social. Introducción a la sociología comprensiva. Buenos Aires: Paidós. 
Bibliografía $\quad$ Scitovsky,Tibor. (1986). Frustración de la riqueza. La satisfacción humana y la insatisfacción del consumidor. México: Fondo de Cultura Económica.

Seligaman, Martin, Railton, Peter, Baumeister, Roy, y Sripada, Chandra. (2016). Homo Prospectur. Nueva York: Oxford University Press.

Sen, Amartya. (1999). Development and Freedom. Oxford: Oxford University Press. 